\title{
SPIKE PENETRATION IN BLAST-WAVE-DRIVEN INSTABILITIES
}

\author{
R. P. DRAKE \\ Atmospheric, Oceanic, \& Space Science, and Applied Physics, University of Michigan, 2455 Hayward Street, Ann Arbor, MI 48109, USA; rpdrake@ umich.edu \\ Received 2011 April 18; accepted 2011 November 22; published 2011 December 22
}

\begin{abstract}
The problem of interest is the unstable growth of structure at density transitions affected by blast waves, which arise in natural environments such as core-collapse supernovae and in laboratory experiments. The resulting spikes of dense material, which penetrate the less dense material, develop broadened tips, but the degree of broadening varies substantially across both experiments and simulations. The variable broadening presumably produces variations in the drag experienced by the spike tips as they penetrate the less dense material. The present work has used semianalytic theory to address the question of how the variation in drag might affect the spike penetration, for cases in which the post-shock interface deceleration can be described by a power law in a normalized time variable. It did so by following the evolution of structure on the interface through the initial shock passage, the subsequent small-amplitude phase of Rayleigh-Taylor instability growth, and the later phase in which the spike growth involves the competition of buoyancy and drag. In all phases, the expansion of the system during its evolution was accounted for and was important. The calculated spike length is strongly affected by the drag attributed to spike tip broadening. One finds from such a calculation that it is not unreasonable for narrow spikes to keep up with the shock front of the blast wave. The implication is that the accuracy of prediction of spike penetration and consequent structure by simulations very likely depends on how accurately they treat the broadening of the spike tips and the associated drag. Experimental validation of spike morphology in simulations would be useful.
\end{abstract}

Key words: hydrodynamics - instabilities - ISM: supernova remnants - shock waves - supernovae: general

Online-only material: color figures

\section{INTRODUCTION}

Experiments, simulations, and observations in nature have long examined the behavior of the Rayleigh-Taylor (RT) instability (Rayleigh 1900; Taylor 1950), driven by the buoyancy of one fluid layer relative to another. Unstable buoyancy can develop by heating of a fluid layer that is deeper within a gravitational potential well, by the deceleration of a denser fluid layer against a less dense layer, or by other means. More recently, the study of systems driven by shock waves made evident the Richtmyer-Meshkov (RM) process (Richtmyer 1960; Meshkov 1969; not strictly speaking an instability), in which the deposition of vorticity as a shock wave crosses an interface leads the interface to develop structure over time. A blast wave is a shock front followed by a deceleration, produced when a shock wave that is no longer sustained is overtaken by a rarefaction. When a blast wave encounters transitions in a fluid where the density decreases, the resulting unstable dynamics is a combination of RM and RT. Such systems are extremely common in astrophysics, where blast waves are produced by any strong but temporary release of energy and where they have many opportunities to encounter transitions in fluid density. Perhaps the archetypical example is the blast wave produced in a core-collapse supernova (SN), which proceeds to cross several such transitions in density as it traverses the stellar envelope. Here we are concerned with a specific and mysterious aspect of blast-wave-driven (BWD) instabilities, known as "spike penetration."

In the jargon of interface instabilities, spikes are the regions of denser material that penetrate beyond the initial interface into the less dense material. These are contrasted with bubbles, which are the converse. The nomenclature arises from RT processes in Earth's gravitational field, where the bubbles rise and the spikes fall. The equations that describe the spikes and bubbles are identical, but their nonlinear structure is not. The spikes are of greater interest in certain contexts. In core-collapse $\mathrm{SNe}$, they carry the inner material outward. If they penetrate far enough, spikes from inner layers can in principle deliver material into the structures produced by instabilities at outer interfaces, as is seen for example in the modeling of Fryxell, Arnett, and Muller (Arnett et al. 1989; Fryxell et al. 1991; Muller et al. 1991) but not in the different models of Kifonidis et al. (2000, 2003, 2006). In present-day experiments at high energy density, measurements of spike structure are far more accurate than those of bubble structure because the spikes are formed by more dense material in a less dense background. These considerations motivate our focus here on the spikes as such.

Early experiments with RM processes in compressible flows involved the study of systems having small changes in density (low "Atwood" number) and/or weak shock waves (see Jacobs \& Sheeley 1996 and references therein). In early work at high Mach number, there was some speculation that RM spikes would never prove able to overtake the driving shock wave (Dimonte et al. 1996). In contrast to these expectations, RM at high Mach number soon proved able to produce spikes that overtook and distorted the shock wave itself (Glendinning et al. 2003). In retrospect, at least for some classes of initial conditions, this is strictly a matter of the relative velocities at which the spikes and the shock advance from the location where one would find an unperturbed interface (the "mean interface" location). This is clearly explained, and applied to astrophysical observations of certain supernova remnants (SNRs), in work by Miles (2009). Pure RT instabilities, in contrast, develop at decelerating interfaces in the absence of shock waves so that there is no interaction of spikes with a shock.

In BWD instabilities, there is the potential for the spikes and the shock front to interact. However, no simulation to date has (to my knowledge) produced a spike that overtook the shock front, with the exception of (at least) one unpublished 
two-dimensional simulation in which overtaking occurred only on axis (Aaron Miles 2009, private communication). In contrast, two experimental studies have seen evidence of spikes that overtake shock waves (Drake et al. 2004; Kuranz et al. 2010). This is not, however, the only difference. The morphology of the spikes (i.e., their lateral structure) differs greatly among simulations and even among experiments studying BWD instabilities. From comparing the simulations, it is not clear to what extent the differences among them arise from the behavior of the numerical scheme at the grid scale, from differences in equations of state, or from other causes. In addition, because the motions of the spike material relative to the surrounding fluid are very subsonic, large differences in structure may result from small variations in vorticity (in either simulations or experiments).

The variation in one aspect of spike morphology might plausibly account for the variations in spike penetration. This is the amount of drag produced by the spike tip. In classic RT systems (Jacobs \& Catton 1988), the spike tip develops a broad, mushroom-like head in consequence of lift forces generated by shear flows near the tip. In the strongly nonlinear phase, the evolution of spike length is determined by the competition between buoyant forces and drag forces (see Dimonte 2000 and references therein). The drag forces in turn depend upon the projected area of the mushroom tip (and also in principle upon its shape). All else being equal, spikes with smaller spreading near the tip would be expected to penetrate further. My goal here is to quantitatively explore the implications. Unfortunately, the required calculation is not trivial.

In order to address the connection of spike penetration and spike tip broadening, we will consider here a description of the dynamics of evolving spikes, aimed at addressing the questions just raised. Our approach involves some limitations and simplifications. The analysis here is planar, as are the relevant experiments to date. We will divide the evolution into two phases. The first phase will be a linear growth phase, which will be taken to last until the amplitude of the perturbation reaches $10 \%$ of its wavelength. After this, we will treat the evolution according to a standard buoyancy-drag model. This approach leaves out the transition of the evolution from strictly linear to strongly nonlinear, during which, for example, the harmonic content of the perturbations increases and the growth rate decreases (Remington et al. 1995). This might result in some difference in the location of the spikes but will not affect their terminal velocity or whether a regime exists in which they can overtake the forward shock, which is our principal interest here.

We will consider only the growth of the spikes beyond the location of the mean interface. We will show that a power-law deceleration of the interface is a sensible model and will use this to produce and analyze the self-similar structure of the material between the forward shock and the mean interface. Under certain assumptions, one could also produce a self-similar model of the material between the reverse shock and the interface, following Chevalier (1982), and could treat both bubbles and spikes. However, as the spikes are more accurately observed in experiments and of substantial interest in core-collapse $\mathrm{SNe}$, this will be our focus here.

Recent work has identified one other mechanism that can affect bubble or spike penetration in RT driven by a steady or slowly varying acceleration. This is the accumulation of vorticity within the structure. This has been observed in simulations (Glimm et al. 2002; Ramaprabhu et al. 2006; Betti \& Sanz 2006; Wei \& Livescu 2011) and in experiments (Wilkinson \& Jacobs
2007). None of this work has explored the case of the steadily decreasing acceleration encountered in BWD instabilities, for which there will be less accumulation of vorticity in the structures. Thus, the potential importance of vorticity accumulation for this case is unclear. If this effect were important, it ought to be present in the simulations of BWD cases as well. Also, in the context of the present discussion, this effect is the opposite of that needed to explain the difference between the existing simulations and experiments. Correspondingly, we focus here on the question raised above, seeking to quantify the effects that spike tip drag may have on spike penetration.

Finally, it is worthwhile to state that the applicability of the present analysis to thermonuclear $\mathrm{SNe}$ is at best uncertain. The thermonuclear burning does produce hot, buoyant matter that drives RT instabilities. However, white dwarf stars do not initially contain composition interfaces within their volume, and in addition the explosion involves the release of energy throughout the star rather than at its core, so it seems unlikely that the development of structure in the explosion can be accurately described as a BWD instability at composition interfaces. That said, if the speculation of Miles (2009) regarding the potential that the structure is strongly affected by initial conditions is correct, then some of the discussion here regarding the interplay of RM and RT will be relevant.

\section{THEORETICAL CONTEXT}

Here we discuss the theoretical models used below and the constraints on their validity.

Buoyancy-driven, unstable hydrodynamic processes often drive the growth of structure in an initially stratified medium. In general, modulations in the density, relative to the background, stratified state, are unstable when the gradients of pressure and entropy are parallel (see chap. 1 of Landau \& Lifshitz 1987 or Drake 2005). This implies, for the case generally described as the RT instability, that such modulations are unstable in systems having opposed gradients of density and pressure. These are the cases of specific interest here. In such cases, the background pressure gradient creates a background acceleration. In the lab frame this acceleration is $-\nabla p_{o} / \rho_{o}$, where the background pressure and density are $p_{o}$ and $\rho_{o}$, respectively. In the non-inertial frame of the interface or of the shock, the acceleration, $\mathbf{a}$, is of opposite sign so $\mathbf{a}=\nabla p_{o} / \rho_{o}$. This acceleration is generally designated as $\mathbf{g}$, by analogy to gravitational acceleration. (In gravitationally dominated systems it is gravity that establishes the background pressure gradient.) In dynamic systems, phenomena such as the accumulation of matter in a shocked layer establish $\nabla p_{o}$. The stratification of the density typically occurs in layers orthogonal to $\nabla p_{o}$. In general, the stratification may involve a continuous change in density, as in a gravitationally bound hydrostatic atmosphere, or a relatively abrupt change (an interface), as when two regions of distinct composition are in contact. Our focus here will be on the second case, which occurs for example in core-collapse SNe or SNRs as an initial transition region evolves into an abrupt interface preceded by a forward shock and followed by a reverse shock. A similar structure develops after a blast wave crosses the interface between disparate materials in laboratory experiments (Ryutov et al. 1999; Kuranz et al. 2009b). We will refer to the density on the denser side of the interface as $\rho_{1}$, and to the (smaller) density on the less dense side of the interface as $\rho_{2}$.

The fundamental equations used to describe the physical system in the following are the Euler equations, which may 
be written as

$$
\begin{gathered}
\frac{\partial \rho}{\partial t}+\nabla \cdot(\rho \mathbf{u})=0, \\
\rho\left(\frac{\partial \mathbf{u}}{\partial t}+\mathbf{u} \cdot \nabla \mathbf{u}\right)=-\nabla p, \text { and } \\
\frac{\partial p}{\partial t}+\mathbf{u} \cdot \nabla p=-\gamma p \nabla \cdot \mathbf{u},
\end{gathered}
$$

where $\rho$ is mass density, $\mathbf{u}$ is fluid velocity, $p$ is pressure, and $\gamma$ is related to the equation of state as is described just below. The models of the RT instability used below require only the first two of these equations, and so depend upon their applicability but not upon the equation of state.

The equation of state enters here in determining the compression produced by the shock wave and the post-shock interface velocity, in Equation (5) below. In order to obtain a relatively tractable description of the unstable dynamics, we need to define a relatively simple treatment of the equation of state. Here we define $\gamma$ as a typical polytropic index such that pressure $p$ and internal energy $\epsilon$ are related as $\epsilon=p /(\gamma-1)$ and $p \propto \rho^{\gamma}$. For a fully ionized plasma state with negligible radiation pressure, $\gamma$ approaches $5 / 3$. If the radiation pressure is dominant, $\gamma=4 / 3$. In an actively ionizing material, $\gamma$ is near $4 / 3$ but 1.4 is often a better value. As a given system evolves among these states, in core-collapse $\mathrm{SNe}$ or in laboratory experiments after the initial ionization, the changes in $\gamma$ are smooth and gradual. Here we take $\gamma$ to be constant within any given material, noting that only phase transitions or strong, anomalous changes in $\gamma$ with the parameters could change the conclusions reached here regarding the question of interest. See also Ryutov et al. (1999) on this point.

The Euler equations are applicable when processes not included in them do not alter the dynamics and for nonrelativistic speeds. They may apply to ionizing matter, ionized matter, matter in which the radiation pressure is dominant, and simple degenerate matter. They may fail, for example, to accurately describe the interface behavior if radiation energy transport removes a significant fraction of the energy from the shocked matter, or if viscous dissipation or viscous momentum transport is significant. They can reasonably be applied to corecollapse $\mathrm{SNe}$ and to laboratory experiments in which similar structures are driven by strong shock waves, as is discussed by Ryutov et al. (1999), and also to a wide range of other astrophysical phenomena as seen in many simulation studies. Working with these equations when they apply and when the equation of state does not depart too greatly from $\gamma$-law-gas behavior provides conclusions whose degree of quantitative accuracy depends upon the impact of the neglected physical processes and upon how close the equation of state is to the model just described.

Within the context of the Euler equations, the validity of the discussion that follows also requires that the blast wave that drives the dynamics at the interface must be stable. If $\gamma$ of either shocked fluid becomes small enough, the density jump at the shock front can become large enough that the thickness of the blast wave becomes small on the scale of relevant transverse dimensions. In this case coupling of waves on the shock with those on the rarefaction can lead to a variant of the Vishniac instability (Vishniac 1983; Vishniac \& Ryu 1989; Doss 2011), causing modulations of the entire layer. For the specific case of non-radiating blast waves, Vishniac \& Ryu (1989) conclude that this threshold is approximately $\gamma \leqslant 1$.1. In addition, a postshock layer could become unstable if radiative losses cause the shell of shocked material to become thin (Liang \& Keilty 2000), even if the value of $\gamma$ as defined above was large.

\section{ONE-DIMENSIONAL MOTION}

\subsection{Initial Interface Dynamics}

In the systems of interest, there will be some initial modulation on the interface and/or on the shock front that traverses it. The shock will be a very strong shock and will move from higher density to lower density as it crosses the interface. The relevant one-dimensional dynamical analysis, used below, is developed in Drake (2006). The details of the shock interaction with a modulated interface in this case set the stage for the subsequent evolution. It will be most useful to work in the "lab frame," in which the upstream fluid is initially at rest. Before it reaches the interface, the shock is of velocity $u_{s 1}$ in the denser material, so that the post-shock fluid velocity is $u_{p 1}=2 u_{s 1} /(\gamma+1)$ and the post-shock sound speed is $c_{1}=u_{p 1} \sqrt{\gamma(\gamma-1) / 2}$. When the shock wave emerges into a second material of much lower density, the interface releases at a speed approaching $u_{\max }=u_{p 1}+2 c_{1} /(\gamma-1)$. In terms of $u_{s 1}$, this is

$$
u_{\max }=\frac{2 u_{s 1}}{(\gamma+1)}\left(1+\sqrt{\frac{2 \gamma}{(\gamma-1)}}\right),
$$

so that the post-shock interface moves faster than $u_{s 1}$ for $\gamma<4.2$. The consequence is that the modulations on the interface become inverted and compressed during the shock passage. (Only for small enough density drops, not of interest here (Drake 2006), does the modulation fail to invert during shock passage.) The actual interface velocity $u_{\text {int }}$ (again ignoring small effects of small modulations) is determined by the balance between the pressure of the adiabatic rarefaction of the denser material and the post-shock pressure in the less dense material, $p_{\text {int }}$. The equation describing this balance is written as

$$
\begin{aligned}
p_{\text {int }} & =\frac{2}{(\gamma+1)} \rho_{1} u_{s 1}^{2}\left(1-\frac{\gamma-1}{2} \frac{\left(u_{\text {int }}-u_{p 1}\right)}{c_{1}}\right)^{2 \gamma /(\gamma-1)} \\
& =\frac{\gamma_{2}+1}{2} \rho_{2} u_{\text {int }}^{2}
\end{aligned}
$$

where the polytropic index of the (shocked) less dense material is $\gamma_{2}$, and $\rho_{1}$ and $\rho_{2}$ remain the initial, unshocked densities of the more dense and less dense materials, respectively. This equation can be solved for $u_{\text {int }}$, but not in any convenient form. Examining its solutions, one finds that $u_{\text {int }}$ is typically within about $25 \%$ of $1.6 u_{s 1}$ and is not strongly sensitive to any of the other parameters so long as $\rho_{1} / \rho_{2}$ is of order five or larger.

A specific modulation is compressed by a factor of $(\gamma+1) /(\gamma-1)$ by the shock but is also stretched during the inversion as the shock passes. If the initial peak-to-valley amplitude is $2 a$ so that the shock-transit time is $\Delta t=2 a / u_{s 1}$, then the amplitude of the inverted modulations after the shock passes, $a_{o}$, is approximately given by $2 a_{o} \sim\left(1.6 u_{s 1} \Delta t-2 a\right)=1.2 a$. The result is that a modulation of initial approximate amplitude $-\left(a_{o} / 0.6\right) \cos (k x)$, where $k$ is the spatial wavenumber, becomes a modulation of amplitude $a_{o} \cos (k x)$ as the shock reaches its end. (Here we ignore the introduction or removal of harmonic components as shock passage occurs.) If we define time $t=t_{s}$ 


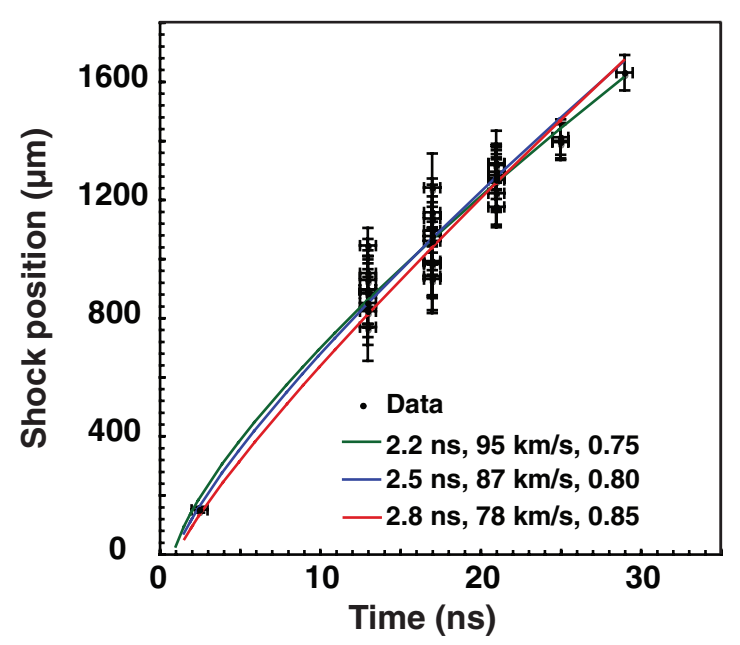

Figure 1. Shock position data from experiments, compared to power-law models. In each case $\alpha$ is determined by a least-squares fit for specific values of $t_{s}$ and $u_{s 2 o}$. The legend shows $t_{s}, u_{s 2 o}$, and $\alpha$. The corresponding values of $t_{1}$ are $1.2,1.4$, and $1.6 \mathrm{~ns}$.

(A color version of this figure is available in the online journal.)

as the moment the shock crosses the mean interface, then the modulation has the shape $a_{o} \cos (k x)$ at time $t_{\delta} \approx t_{s}+\left(a / u_{s 1}\right)$. Given that our concern is with much longer evolution times, we can take $t_{\delta}=t_{s}$ for our work here. The specific parameters of our reference laboratory experiment confirm this. For characteristic (Miles et al. 2004; Drake et al. 2004; Kuranz et al. 2009a) experimental parameters $\left(t_{s} \sim 2.5 \mathrm{~ns}, u_{s 1} \sim 50 \mathrm{~km} \mathrm{~s}^{-1}\right.$, and $a \sim 2.5 \mu \mathrm{m}$ ), one has $a_{o} \sim 1.5 \mu \mathrm{m}$ and $t_{\delta} \sim t_{s}+0.05 \mathrm{~ns}$, with $t_{s} \sim 2.5 \mathrm{~ns}$. This confirms that it will suffice here to take $t_{\delta}=t_{s}$.

Two related items are worth noting. First, the initial shock velocity in the low-density fluid is $u_{s 2 o}=(\gamma+1) u_{\text {int }} / 2$. For $u_{\text {int }}$ as estimated above and $\gamma=1.4$, this is $u_{s 2 o} \sim 1.9 u_{s 1}$. Second, practical simulations do not resolve the shock well enough to follow the dynamics just described. To do so would require accurate resolution of the multidimensional behavior of the shock wave as it propagated through the modulations of the interface, which are themselves often a small fraction of the corresponding wavelength. This translates into a need for at least thousands of zones per wavelength (and perhaps much more), a substantial challenge in three dimensions. The evaluation of the response of the interface at early times by practical simulations is correspondingly suspect.

\subsection{Interface Deceleration}

In our systems of interest, the interface immediately begins to decelerate as the shock wave in the lower-density region accumulates mass. This effect is negligible during the short time from $t=t_{s}-\Delta t / 2$ to $t=t_{s}+\Delta t / 2=t_{\delta}$, but dominates the longer-term behavior. In the experiments, the observed shock positions included considerable variability that is not understood in detail. A likely speculation is that variations in the actual alignment of the target to the laser beams cause variations in average laser ablation pressure. Figure 1 shows relevant experimental data, with error bars corresponding only to the well-understood sources of variability. One can treat the deceleration as a power law in time, as for example does Chevalier (1976). The figure shows three power-law models of these data. The form of these models is $R_{s}=R_{o} \tau^{\alpha} \mu \mathrm{m}$, where
$R_{S}$ is the shock position and

$$
\tau=\frac{t-t_{o}}{t_{s}-t_{o}}=\frac{t-t_{o}}{t_{1}},
$$

where the time that the fitted curve reaches $r=0$ is $t_{o}$. Note that this time does not correspond to an actual shock location, but rather serves to define the curve that approximates the shock position after the shock crosses the interface. In addition, Equation (6) defines $t_{1}$, which will be useful below. One can see in the figure that the experimental data in no sense demand such a power-law dependence, but are reasonably consistent with such a model. The point of using such a model is not to fit any specific physical system, but rather to become able to explore the interplay of spike tip broadening and spike penetration. We will use the nominal, central fit for specific applications below.

The fit just described also implies an initial shock velocity within the lower-density material (shocked foam in the reference experiment), labeled above $u_{s 2 o}$. This is

$$
u_{s 2 o}=\frac{\alpha R_{o}}{t_{1}},
$$

where $u_{s 2 o}$ is connected with the initial shock front velocity in the denser material, $u_{s 1}$, as described above. In developing the fits shown in Figure 1, $u_{s 2 o}$ was constrained to be consistent with the value of $u_{s 1}$ corresponding to the shock breakout time.

As the shock wave propagates into the low-density material and decelerates, the shocked material develops density structure for two reasons. First, the pressure gradient produced by the deceleration implies that initially shocked material must expand adiabatically as the shock moves beyond it. Second, the entire shocked layer gradually expands as the post-shock pressure slowly decreases as the shock slows. These effects cause the density near the interface to be lower than the immediate postshock density. We can solve for the density profile on the assumption that the shock wave has a power-law behavior and propagates into a material of constant density, which allows us to treat the shocked, low-density material as a self-similar system. This is essentially Parker's 1963 model of an expansion-driven outer shock (Parker 1963). We need to understand this expansion in order to account for its influence on the observed instability growth.

As a similarity variable, one can use $\xi=r / R_{s}$, where $r$ is position, and we work in one dimension here. In this case the Euler equations for density, momentum, and pressure can be recast as ordinary differential equations, as for example in Drake (2006). Here the relation of the physical variables $\rho, u$, and $p$ to the corresponding similarity variables $\Omega, U$, and $P$ is $\rho=\rho_{2} \Omega(\xi), u=\dot{R}_{s} U(\xi)$, and $p=\rho_{2} \dot{R}_{s}{ }^{2} P(\xi)$. The boundary conditions at the shock front are strong-shock conditions, so that the post-shock density, velocity, and pressure there are $\rho=\rho_{2}(\gamma+1) /(\gamma-1), u=2 \dot{R}_{s} /(\gamma+1)$, and $p=2 \rho_{2} \dot{R}_{s}^{2} /(\gamma+1)$, respectively. Figure 2 shows the resulting profiles for $\gamma=1.4$ and $\alpha=0.8$, which are reasonable for the experimental case. The values are normalized to the boundary conditions just given. At any given time, the interface-driven shock traces out as much of this structure as is necessary to conserve mass, so that a larger fraction of the profiles shown in the figure is present at later times. Eventually, the pressure at the interface drops below the value required for the self-similar solution, and the interface stops and reverses direction. The self-similar model then no longer applies and the expansion of the shocked material becomes more rapid. 


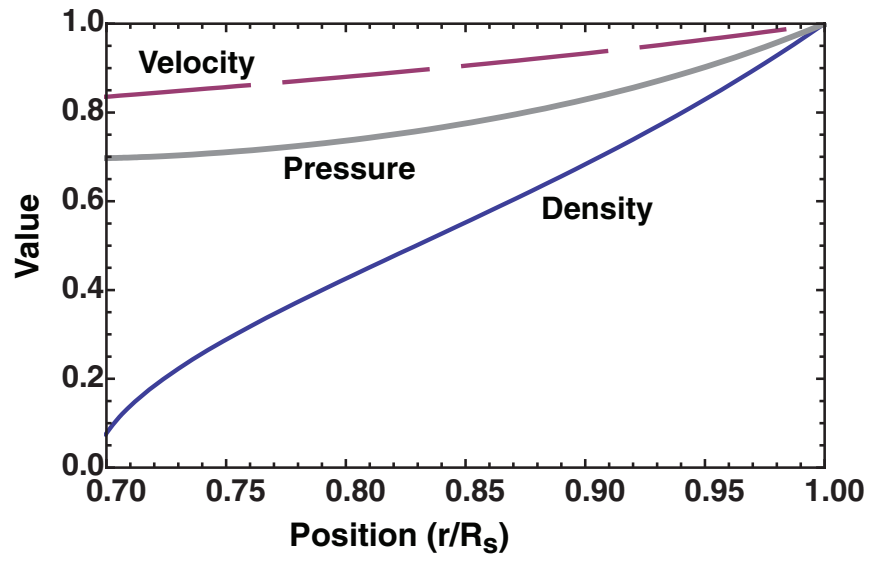

Figure 2. Self-similar profiles of the shocked, low-density material for a powerlaw shock deceleration such that $R_{S} \propto \tau^{0.8}$

(A color version of this figure is available in the online journal.)

The conservation of mass can be expressed as

$$
\rho_{2}\left(R_{s}-R_{o}\right)=\int_{R_{p}}^{R_{s}} \rho(r) d r
$$

where $R_{p}$ is the interface (or "piston") position. One can integrate a profile like that of Figure 2 to find $R_{p}$ as a function of $R_{o}$ and $R_{s}$. For $\gamma=1.4$ and $\alpha=0.8$, as above, the corresponding function is fit over the range of interest, to within $1 \%$ accuracy, by

$$
\frac{R_{p}}{R_{S}}=0.717+0.547\left(\frac{R_{o}}{R_{S}}\right)-0.454\left(\frac{R_{o}}{R_{S}}\right)^{2}+0.193\left(\frac{R_{o}}{R_{S}}\right)^{3} .
$$

Given the power-law dependence of $R_{s}$ on $\tau$, one can fit $R_{p}$ as a power of $\tau$ for specific applications. For the experiments cited above, one finds $R_{p}=R_{o} \tau^{\alpha_{p}}=150 \tau^{0.708}$ in $\mu \mathrm{m}$. Figure 3 compares this fit to a numerical solution of Equation (8). The fit is accurate to $3 \%$. The corresponding acceleration of the interface is $\alpha_{p}\left(\alpha_{p}-1\right) R_{o} t_{1}^{-2} \tau^{\alpha_{p}-2}=-15.8 \tau^{-1.29} \mu \mathrm{m} \mathrm{ns}^{-2}$. We will use this below.

\subsection{Expansion of Shocked Matter}

The shocked matter expands as the shock decelerates. One consequence is that the length of a spike or bubble increases both because of unstable growth and because of the expansion. We will refer to the spikes henceforth but a similar analysis could be developed for the bubbles, subject to understanding the spatial profiles on the denser side of the interface. Locally, the expansion occurs adiabatically when the pressure decreases as material moves further from the shock. However, because lateral pressure gradients smooth rapidly in the subsonic region where the instability grows, the spikes and bubbles are at the same pressure (at any given position along their length) and expand corresponding to equal changes in pressure. The increase of spike length includes contributions from both the length increase due to expansion and the length increase due to the instability. One can describe this, following Miles (2009), as

$$
\frac{d h}{d t}=u_{\mathrm{inst}}+\eta h
$$

where the length of the spike (the displacement of its tip from the mean interface location) is $h, u_{\text {inst }}$ is the rate of increase

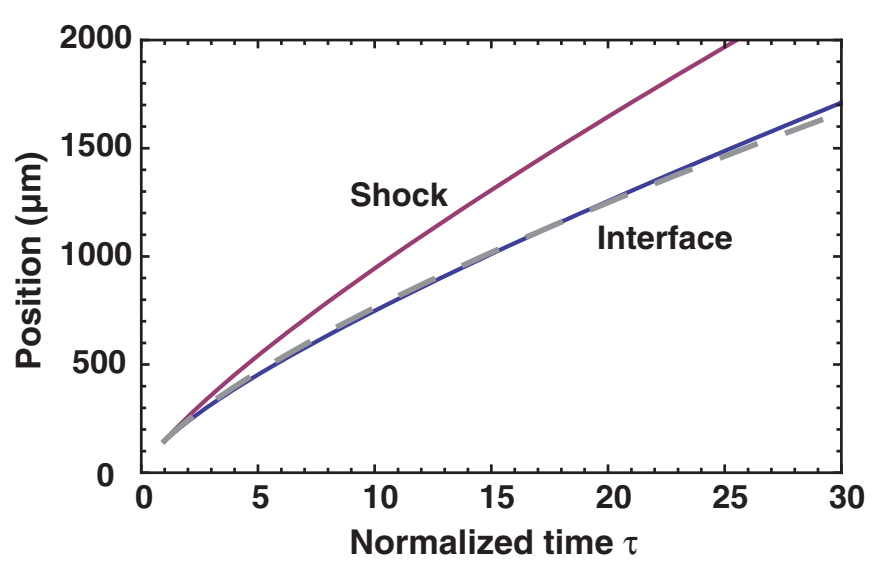

Figure 3. Shock position and interface position. The dashed line shows the power-law fit to interface position discussed in the text. The shock position corresponds to $\gamma=1.4$ and $\alpha=0.8$. The interface fit has $\alpha_{p}=0.708$.

(A color version of this figure is available in the online journal.)

in the spike length due to the instability, and $\eta$ is defined for one-dimensional expansions as

$$
\eta=\frac{1}{h} \int_{R_{p}}^{R_{t}} \frac{\partial \ln v}{\partial t} d r
$$

where $R_{t}$ is the spike tip location, so $h=R_{t}-R_{p}$, and $v$ is the specific volume $(=1 / \rho)$, so $\partial(\ln v) / \partial t$ is the fractional rate of expansion. The fractional rate of expansion can be found, from the continuity equation, Equation (1), to be

$$
\frac{\partial \ln v}{\partial t}=\frac{\partial u}{\partial r}+u \frac{\partial \ln \rho}{\partial r}
$$

for planar expansions for which $u=0$ in the frame of reference of interest, which in this case is the frame of the interface. (In other frames of reference, one finds a total derivative on the left-hand side.)

The instability growth is best analyzed relative to the location of the interface in the absence of instability (designated the mean interface), and thus in the non-inertial frame of reference moving with the interface. Thus, one seeks to evaluate Equations (10), (11), and (12) in this frame. Sufficiently close to the interface $u \sim 0$ so $\eta \sim \partial u / \partial r$ and the analysis of Miles (2009) follows. We refer to this limit as the Miles approximation below. Otherwise, we can use the self-similar solution described above to evaluate $\eta$ as a function of interface position and spike position. In terms of this solution, $u=\dot{R}_{s}\left(U(\xi)-U\left(\xi_{p}\right)\right)$, $\dot{R}_{s} / R_{s}=\alpha /\left(t_{1} \tau\right)$, and

$\eta=\frac{\alpha}{t_{1} \tau} \frac{1}{\left(\xi_{t}-\xi_{p}\right)} \int_{\xi_{p}}^{\xi_{t}}\left(\frac{\partial U(\xi)}{\partial \xi}+\frac{\left(U(\xi)-U\left(\xi_{p}\right)\right)}{\Omega(\xi)} \frac{\partial \Omega(\xi)}{\partial \xi}\right) d \xi$,

where $\xi_{t}$ is the similarity variable corresponding to $R_{t}$.

Figure 4 shows the argument of the integral in Equation (13) as solid lines. One proceeds as follows to find the factor $\eta$ at any given moment. First, one must integrate these curves from $\left(\xi-\xi_{p}\right)=0$ to $\left(\xi_{t}-\xi_{p}\right)$ and then divide the result by $\left(\xi_{t}-\xi_{p}\right)$, thus obtaining an average of the values shown from the interface to the spike tip. Second, to obtain properly scaled physical units of inverse time, one must multiply the result by $\dot{R}_{s} / R_{s}=\alpha /\left(t_{1} \tau\right)$. Figure 5 shows the full range of values of the 


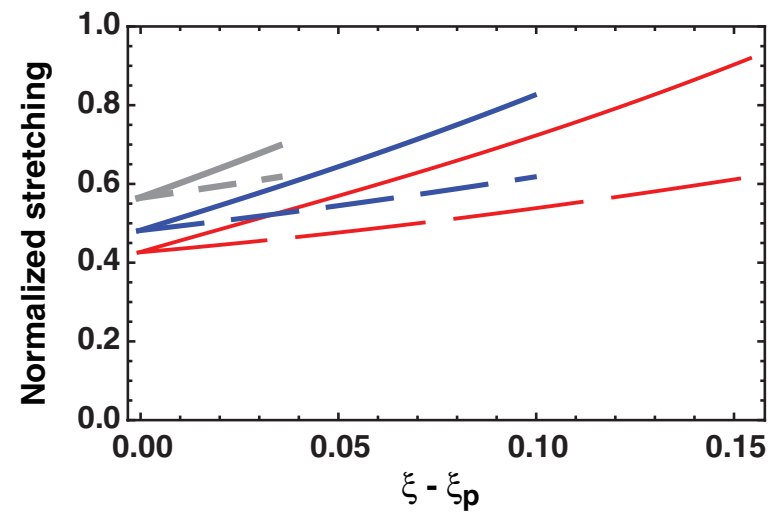

Figure 4. Relative values of $\partial(\ln v) / \partial t$ are shown against normalized distance from the interface location, extending in each case to the shock. These are evaluated for a power-law shock position having $\alpha=0.8$ in a fluid with $\gamma=1.4$. From bottom to top, these correspond to $\xi_{p}=R_{p} / R_{s}$ of $0.85,0.90$, and 0.96 . In each case the dashed curve shows the Miles approximation and the solid curve shows the full result.

(A color version of this figure is available in the online journal.)

average just described. For calculations that combine unstable behavior with stretching, one can interpolate the value of $\eta$ from such results.

In working with Equation (10) one must evaluate the rate of increase of the spike length due to the expansion and also due to the instability. It is worthwhile to discuss this further, as one may wonder what effect the expansion itself may have on the instability. We note that the expansion stretches both the bubbles and the spikes, but does not change the amount of fluid that has penetrated beyond the mean interface location into the other fluid (the amount of interpenetration). The instability equations that govern the interpenetration of the fluids, in both the linear and nonlinear regimes, do not depend explicitly on density, but rather only on the Atwood number and hence on the density ratio at the interface. Equation (12) implies that the rate of stretching is continuous across the interface, and thus that the density ratio remains constant. (One also observes this in simulations.) The instability equations used below are derived under the assumption of incompressibility, but because the evolution of the spikes and bubbles is very subsonic, these equations should remain accurate here. As a result, the time dependence of the unstable growth can be taken directly from the solutions of the instability equations, without needing to further consider the effects of expansion. This conclusion would have to be modified if we intended to follow the evolution of the modal content of the interface modulations as the amplitude increases, as the stretching does alter this. However, we will not need to account for this effect because individual spikes or bubbles of significant amplitude act largely independently (Dimonte 2000; Kuranz et al. 2009a).

\section{EVOLUTION OF TWO-DIMENSIONAL STRUCTURE}

\subsection{Linear Phase}

Now we turn to the first phase of the evaluation of $u_{\text {inst }}$, while the amplitude of the modulations is small. The passage of the shock over the modulated interface inverts, compresses, and stretches the modulations, as discussed above. In addition, it produces lateral velocity modulations on the interface (a process often referred to as the deposition of vorticity). Here we consider the specific case of a mean interface in the $x-y$ plane. In the absence of further acceleration of the interface, the modulations

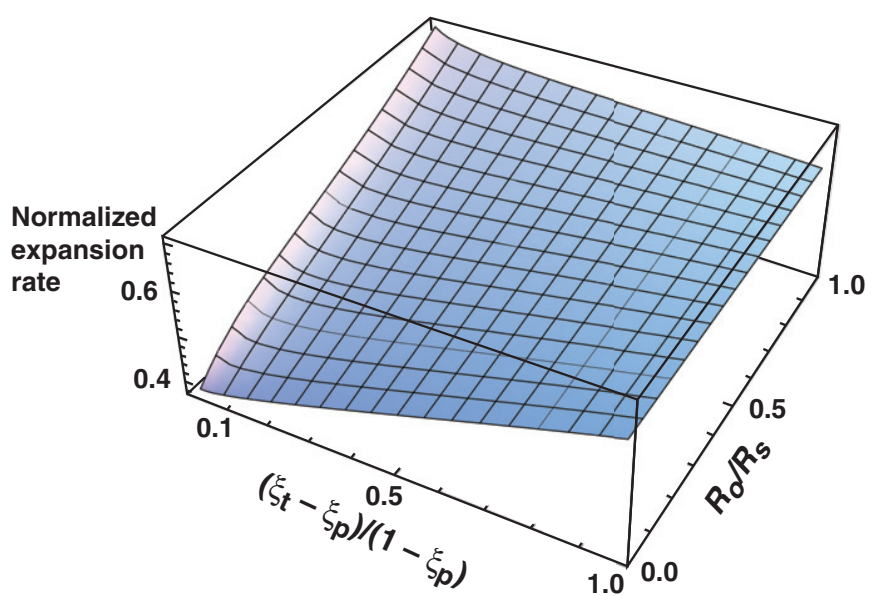

Figure 5. Values of $\eta t_{1} \tau / \alpha$ evaluated from Equation (13), for a system with $\alpha=0.8$ and $\gamma=1.4$. The results are displayed against $R_{o} / R_{s}$, which implies $\xi_{p}$ as discussed above, and the spike length as a fraction of the interface-to-shock distance, $\left(\xi_{t}-\xi_{p}\right) /\left(1-\xi_{p}\right)$.

(A color version of this figure is available in the online journal.)

would evolve via the RM process. We take the initial velocity in the $z$-direction, $w$, immediately after shock passage, to be that corresponding to the Richtmyer model of this process, so that

$$
w\left(t=t_{s}, z=0\right)=w_{i}=k a_{o} A u_{\mathrm{int}} \cos (k x),
$$

where $k$ is the wavenumber of the modulation, $z=0$ is the location of the mean interface in its accelerating frame of reference, and $A$ is the post-shock Atwood number of the interface, given by $\left(\rho_{2}-\rho_{1}\right) /\left(\rho_{2}+\rho_{1}\right)$. Here we define $A$ as positive and note again that the interface inverts during shock transit for cases of interest. There is some support in the literature for the use of an average of the pre-shock and postshock modulation amplitudes in this expression. This arose from the examination of simulation results (Meyer \& Blewett 1972) and experiments (Dimonte \& Remington 1993; Dimonte et al. 1996), which show that this is a better model than the use of the post-shock amplitude without consideration of the shock-transit dynamics discussed above. I suggest that in reality the behavior may be that corresponding to Equation (14), evaluated with the actual post-shock amplitude including both compression and stretching during shock transit. None of the prior literature has considered this stretching in discussing RM. However, sorting this out is not important to address the question that drives the present paper. Dimonte \& Ramaprabhu (2010) provide an extensive review and discussion of the nonlinear evolution of pure RM. In addition, Wouchuk (2001) provides a more general model of the asymptotic growth rate of pure RM from linear theory, beginning with the moment after the shock has crossed the interface, in the context of prior RM theory. However, these works are not relevant here where the interface decelerates immediately following shock passage.

Fundamentally, in the BWD instability, the shock passage creates an initial state for RT. Because the interface decelerates from the moment the shock enters the lower-density material, the further evolution of the modulations takes place under conditions of RT instability. There is no period of time during which one could say the modulations grow by RM effects alone. We proceed here to apply the linear incompressible theory to describe the subsequent RT growth. The fundamental justification for this is that the compressible terms in the fluid equations scale as Mach number squared, and the fastest rate 
of increase of the spike amplitude is of order $1 / 4$ of the sound speed in the shocked, low-density matter. Correspondingly, there is very little compression associated with the instability growth as such and compressible effects should be small. The specific correction terms found in the compressible theory of the RT instability (Mitchner \& Landshoff 1964; Plesset \& Hsieh 1964) are of order $g /\left(k c_{s}^{2}\right)$ as compared to 1 , where the sound speed at the interface is $c_{s}$. The quantity $g /\left(k c_{s}^{2}\right)$ is $\leqslant 0.1$ and decreases with time in the specific case we consider here. The compressible effect that does matter here is the expansion discussed above and accounted for below.

If the initial modulations are in the linear regime, as assumed in the derivation of Equation (14), and as we will assume here, then the corresponding linear RT theory is like that developed by Chandrasekhar (1961). Here, for specific conditions of interest, we quote the results of derivations identical to those he describes. We take the interface to be abrupt, the densities to be constant on either side of the interface, and the viscosity to be negligible. Assuming a $\cos (k x)$ or equivalent $x$-dependence, the differential equation for $\zeta$, the displacement of an element of fluid relative to its initial displacement from the mean interface, is

$$
\rho_{o}\left(\partial_{z}^{2}-k^{2}\right) \partial_{t}^{2} \zeta=-g\left(\partial_{z} \rho_{o}\right) k^{2} \zeta
$$

where $\partial_{q}=\partial / \partial q, \rho_{o}$ is the initial density profile, and in general $g$ is time dependent. Here when $g$ is positive the pressure and density gradients are opposed and instability growth occurs. This equation also holds for $\rho_{o}$ that varies only at the interface. In contrast, the analogous differential equation for $w$ includes a term involving both $\zeta$ and the derivative of $g$ in time, not easily resolved. When $\partial_{z} \rho_{o} \approx 0$ except at the interface, and under boundary conditions such that $\zeta \rightarrow 0$ at $z \rightarrow \pm \infty$, this implies that $\zeta \propto \exp (-k z)$ for $z>\zeta$ and $\zeta \propto \exp (k z)$ for $z<\zeta$.

With the above assumptions and results, the boundary conditions give the general differential equation for $\zeta$,

$$
\partial_{t}^{2} \zeta-A g k \zeta=0
$$

valid for time-dependent $g$. For constant $g$ this has the familiar solutions proportional to $\exp ( \pm \sqrt{A k g} t)$. We consider here the case in which $g$ varies as a power law in time, so that

$$
g=g_{o} \tau^{\beta}
$$

Here, $\tau$ is defined as above for a power-law interface position $R_{p}$, in terms of a shock breakout time $t_{s}$ and a reference time $t_{o}$, whose difference is $t_{1}$. With $R_{p}=R_{o} \tau^{\alpha_{p}}$ as above, and maintaining the required sign for $g$ relative to the above derivations, one has $g_{o}=\alpha_{p}\left(1-\alpha_{p}\right) R_{o} / t_{1}^{2}$.

The displacement of the interface, $\zeta_{o}$, must be the same when approached from either direction. Using the definition of $\tau$ to transform Equation (16) to an equation for $\zeta_{o}(\tau)$, one finds

$$
\partial_{\tau}^{2} \zeta_{o}-d^{2} \tau^{\beta} \zeta_{o}=0
$$

where $d^{2}=\left(A k g_{o} t_{1}^{2}\right)$.

For the case of decelerating blast waves of interest here, one wants a solution to this equation starting at time $t=t_{s}$. The power $\beta$ is $\leqslant 1$ and $g$ starts at a finite value. For $\beta=0$, Equation (18) gives the solution discussed above, as it should. For non-zero $\beta$, the solution involves the modified Bessel functions of the first kind and is as follows:

$\zeta_{o}(\tau)=C_{1} \sqrt{\tau} I_{\frac{1}{\beta+2}}\left(\frac{2 d}{\beta+2} \tau^{\frac{\beta}{2}+1}\right)+C_{2} \sqrt{\tau} I_{\frac{-1}{\beta+2}}\left(\frac{2 d}{\beta+2} \tau^{\frac{\beta}{2}+1}\right)$,

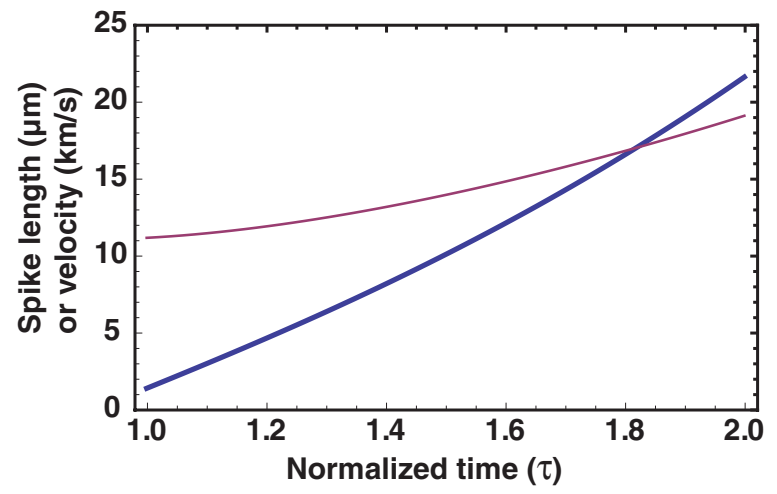

Figure 6. Evolution of modulation amplitude (thick, blue) and velocity (thin, red) under the influence of a power-law deceleration.

(A color version of this figure is available in the online journal.)

in which $I_{\chi}$ is the modified Bessel function of the first kind with index $\chi$ and the constants are set to match the boundary conditions. Taking the derivative of this equation gives the equation for the velocity of the interface, which is

$$
\begin{aligned}
w_{o}(\tau)= & C_{1} \tau^{(1+\beta) / 2} I_{\frac{\beta+1}{\beta+2}}\left(\frac{2 d}{\beta+2} \tau^{\frac{\beta}{2}+1}\right) \\
& +C_{2} \tau^{(1+\beta) / 2} I_{-\frac{\beta+1}{\beta+2}}\left(\frac{2 d}{\beta+2} \tau^{\frac{\beta}{2}+1}\right) .
\end{aligned}
$$

We evaluate the result for the specific case of the experiments discussed above. For these experiments, approximate values of the parameters are $A=0.7, k=$ $2 \pi / 71 \mu \mathrm{m}, a_{o}=1.5 \mu \mathrm{m}, u_{\text {int }}=75.9 \mathrm{~km} \mathrm{~s}^{-1}, g_{o}=15.8 \mu \mathrm{m} \mathrm{ns}^{-2}$, $t_{s}=2.5 \mathrm{~ns}, \beta=-1.29$, and $w_{i}=11.3 \mu \mathrm{m} \mathrm{ns}^{-1}$, so that $d=$ 1.4. In this case Equations (19) and (20) become

$$
\begin{aligned}
\zeta_{o}(\tau)= & 471 \sqrt{\tau} I_{-1.41}\left(3.92 \tau^{0.354}\right) \\
& -470 \sqrt{\tau} I_{1.41}\left(3.92 \tau^{0.354}\right) \text { and } \\
w_{o}(\tau)= & 466 \tau^{-0.146} I_{-0.41}\left(3.92 \tau^{0.354}\right) \\
& -465 \tau^{-0.146} I_{0.41}\left(3.92 \tau^{0.354}\right) .
\end{aligned}
$$

These are plotted in Figure 6, in which the thicker curve is $\zeta_{0}$ in $\mu \mathrm{m}$ and the thinner curve is $w_{o}$ in $\mathrm{km} \mathrm{s}^{-1}$.

For this specific case, we evaluate the effect of expansion as discussed in Section 3.3, by evaluating the spike amplitude equation (Equation (10)) using the results for the expansion rate $\eta$ whose normalized values are displayed in Figure 5. Numerical integration of this equation gives the results shown in Figure 7. For this specific case the amplitude corresponding to $10 \%$ of the wavelength is $7.1 \mu \mathrm{m}$. One sees that this is reached quickly, at a time of 1.3 in units of $\tau$. This corresponds to a physical time of $4.3 \mathrm{~ns}$ (the shock breakout was at $2.5 \mathrm{~ns}$ ). During the linear RT phase one sees that the relative effect of the expansion is quite small.

The equations developed here describe the instability growth through its initial, linear phase. Before we turn to the nonlinear phase, we should note that such a phase may not even exist. If the initial modulations of the interface were large enough that their post-shock amplitude were more than about $10 \%$ of their wavelength, their further growth would be nonlinear from the start. 


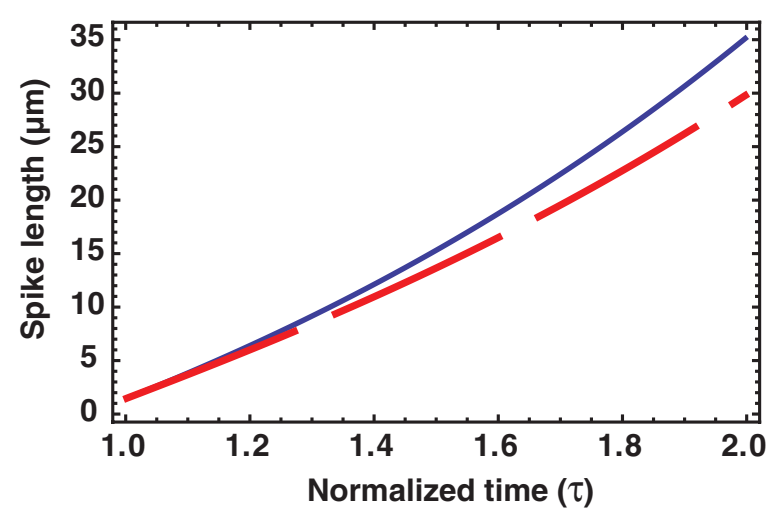

Figure 7. Evolution of modulation amplitude during the linear-theory RT growth phase including (solid, blue) and not including (dashed, red) effects of expansion under the influence of a power-law deceleration.

(A color version of this figure is available in the online journal.)

\subsection{Buoyancy-drag Evolution}

We consider next the evolution of a spike of nonlinear amplitude, falling toward the region of higher pressure. The corresponding basic equation for the evolution of the spike velocity $u$ in the direction of $g$ (taken to be positive), under the simplifying assumption that the density is constant throughout the spike, is

$$
\left(\left(\rho_{1}+\rho_{2}\right) \frac{d u}{d t}-\left(\rho_{1}-\rho_{2}\right) g\right) V=-\left(C \rho_{2} u^{2}\right) A_{s},
$$

in which $C$ is a drag coefficient, $V$ is the volume of the spike, and $A_{s}$ is the area of the spike tip. The various terms in this equation deserve some discussion. The factor of $\rho_{2}$ on the leftmost term in this equation represents the effect of the mass of the less dense fluid displaced by the spike. In general the coefficient on $\rho_{2}$ depends on its shape and might not be unity (see chap. 1 of Landau \& Lifshitz 1987). The buoyancy force, proportional to $g$, might be multiplied by a numerical factor if the mixing of the fluid reduces the density contrast, but can be taken to be unity here, to satisfy the physical constraint that as $\rho_{1}$ approaches zero the spike motion should approach free fall. The drag coefficient $C$ has a value of $2 \pi$ for the cylindrical, three-dimensional (3D) spikes that are relevant here (it is $6 \pi$ for two-dimensional spikes) but may also depend in detail on other factors. Finally, the volume of the spike corresponds to the material that has penetrated beyond the location of the mean interface and the area of the spike tip is the cross-sectional area transverse to the flow. Note that for bubbles the factor of $\rho_{2}$ on the right-hand side would be replaced by $\rho_{1}$. A much more thorough discussion of all these terms may be found in Dimonte (2000).

There are two common limits of the ratio $V / A_{s}$ in the literature, neither of which will apply in the end to the cases of interest here. In the first limit, the spikes or bubbles are simple, cylindrical structures, so that this ratio is the instantaneous amplitude $h$ of the spike (Dimonte 2000). This model has effectively explained a number of experiments (Kuranz et al. 2009a) in which the observed shape of the spikes is more or less cylindrical. In the second limit, bubbles exist over a large range of spatial scales but are observed to have a self-similar structure, so that $V / A_{s}$ is proportional to the bubble width, often designated $\lambda$ and corresponding approximately to $2 \pi / k$. The drag term then is smaller for the larger bubbles, which grow less during the initial phase but eventually reach larger amplitude than the smaller ones. Given a broad spectrum of initial bubbles (or sufficient mode coupling), one can infer that the bubble amplitude grows in time, $t$, as $\alpha_{B} g t^{2}$, where $\alpha_{B}$ is a coefficient of order 0.05 whose exact value has been the subject of much discussion (see Dimonte et al. 2004 and references therein). In such many-mode systems the spikes end up squeezed in between the dominant bubbles at any given time and so do not exhibit the same self-similar behavior. They tend to have an $\alpha_{S}$, defined for spikes by analogy to $\alpha_{B}$ for bubbles, that is larger and that depends (see Dimonte 2000 and references therein) on the density ratio $\rho_{2} / \rho_{1}$.

However, it is unclear whether astrophysical systems resemble either of these limits. Observations of structure attributed to RT instabilities, in the SNR E0102 (Hughes et al. 2000), in the Crab pulsar wind nebula (Hester et al. 1996), and in the Tycho SNR (Warren et al. 2005), seem dominated by distinctly spaced, relatively large-scale structures rather than the sort of finely structured, many-mode RT mix layer seen in some experiments (Dimonte \& Schneider 1996) and some simulations (for example, see Cabot 2006), having very broad modulation spectra as initial conditions. This may reflect the finite duration of the acceleration in the astrophysical systems combined with divergence, which limits the lateral scale of the largest structures that can grow to large amplitude. Alternatively, it may reflect the scale of the structures in the initial conditions. However, in simulations at least the spikes in such systems are not simple cylinders but rather grow "mushroom caps." This can be seen, for example, in simulations of SNRs (Chevalier et al. 1992) and of SN 1987A (Fryxell et al. 1991; Kifonidis et al. 2006).

The physical origin of these mushroom caps is a well-known shear flow effect. The fluid displaced by the spike tips must flow more quickly than the adjacent fluid, lowering the pressure at the spike tips and allowing them to expand laterally. The important physical point here is that these mushroom caps would be expected to increase the drag on the spikes. Qualitatively, it seems clear that larger mushroom caps will produce more drag. Quantitatively, the eddy flow behind the caps will change the drag as would any turbulence. It is difficult to gain insight from published simulation results about the connection between spike tip structure and drag. Most of the simulation work is pure RT or pure RM, and in the BWD work there is no obvious correlation of spike tip shape with penetration (Miles et al. 2004). However, typically the publications show the shape of the structures at few times, and it is the integrated behavior over time that determines the penetration. In one case of pure RT, when increased resolution produces narrower spikes they penetrate further (Calder et al. 2002). It is important to also note that the actual size of the mushroom caps as evaluated by simulations is rather uncertain. The generation of the mushroom caps involves small-scale, subsonic flow and regions of high vorticity, which are not necessarily well resolved or accurately calculated. Certainly different simulations, or even different equation-of-state treatments in the same simulation (Miles et al. 2004), give varying results for the cap size. One must conclude that while the presence and origin of the mushroom caps are clear, their precise structural details and the resulting drag they should produce are not well known.

Here we will deal with this uncertainty by formally defining $A_{s}$ as an effective area-the area of a cylindrical structure whose drag equals that produced by the mushroom cap. We will then express the ratio $A_{s} / V$ as $\psi^{2} / h$, where $\psi=\sqrt{A_{s} / A_{\text {stem }}}$, in which $A_{\text {stem }}$ is the average cross-sectional area of the spike, dominated by the stem but including the mushroom cap so that 


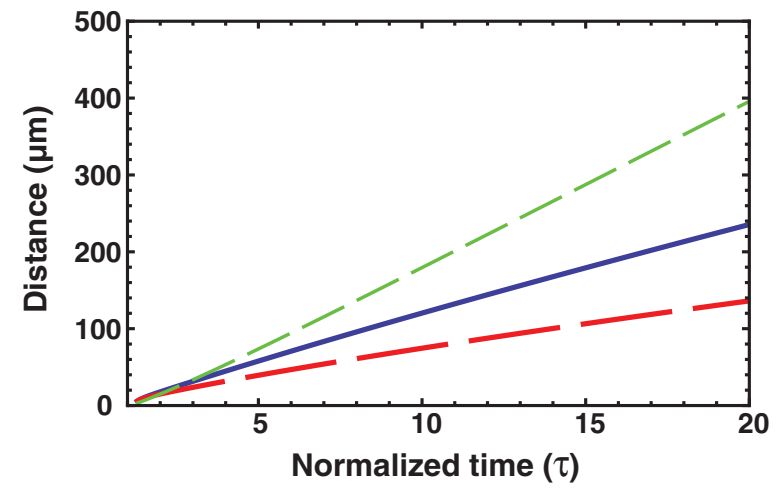

Figure 8. Distance from the mean interface is plotted against $\tau$, for $\psi=1.5$ and other parameters of the reference case $\left(\gamma=1.4, \alpha=0.8, \alpha_{p}=0.71, \beta=\right.$ $\left.-1.29, g_{o}=15.8 \mu \mathrm{m} \mathrm{ns}^{-2}, A=0.7, t_{1}=1.4 \mathrm{~ns}\right)$. The solid blue line is the computed position of the spike tip from the combination of buoyancy drag effects and expansion, the red long-dashed line is without expansion, and the green dashed line is the position of the shock.

(A color version of this figure is available in the online journal.)

the total volume is correct. Thus, $\psi$ is approximately the ratio of the cap radius to the stem radius. It may range in value from one (or perhaps less for pointed spikes as seem to have been observed; Kuranz et al. 2009a) to several. We will use 5 as an upper limit. In general $\psi$ may vary as the spikes evolve in time, but we will treat it as constant to characterize the range of possible behavior. With these definitions, and recognizing that $u=d h / d t$, Equation (23) for 3D spikes becomes

$$
h \frac{d^{2} h}{d t^{2}}+(1-A) \pi \psi^{2}\left(\frac{d h}{d t}\right)^{2}-A g h=0 .
$$

For simplicity we will suppose that $\psi$ is constant and continue to use the previous model that $g=g_{o} \tau^{\beta}$. We also note that, once again, the rate of growth of the spikes does not depend explicitly on density, so that the discussion of the previous section regarding how to include the effects of expansion still applies.

It is also worthwhile to emphasize a difference between the present analysis and most models of pure RT in the literature. The models in the literature (see Ramaprabhu \& Dimonte 2005 and references therein) typically allow spikes or bubbles to grow to nonlinear amplitude and then immediately assign them the asymptotic velocity found by setting the second derivative in Equation (23) equal to zero. However, the transition to this velocity takes increasing time at high Atwood number, as can be seen in Figure 9 of Ramaprabhu \& Dimonte (2005). In the present work, in contrast to the pure RT cases just discussed, we solve for the time-dependent velocity using the buoyancy-drag equation. This would be needed for pure RT for experiments like those of interest and for the many astrophysical systems that have $A>0.5$. It is even more important when the system is BWD because then the asymptotic velocity decreases with time, greatly complicating any attempt to base an analysis on this velocity.

In terms of the variable $\tau$ and other variables defined above, Equation (24) becomes

$$
h \frac{d^{2} h}{d \tau^{2}}+(1-A) \pi \psi^{2}\left(\frac{d h}{d \tau}\right)^{2}-A g_{o} t_{1}^{2} \tau^{\beta} h=0 .
$$

For specific values of the parameters, one can solve this numerically and thus find $u_{\text {inst }}$ as is needed in Equation (10).

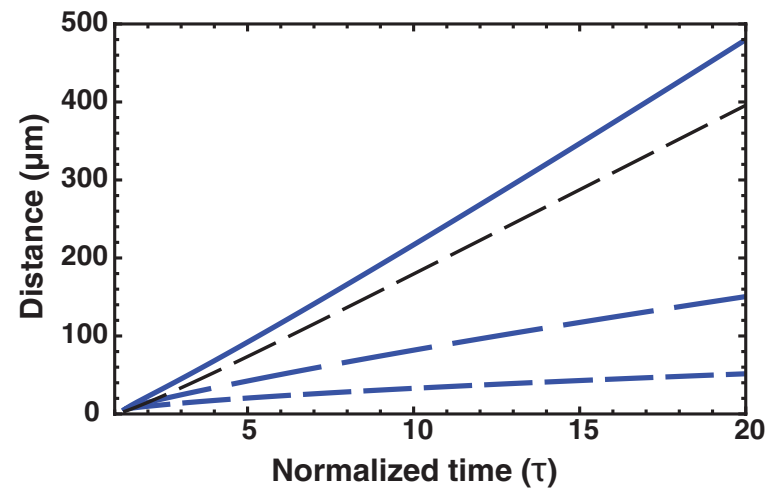

Figure 9. Distance from the mean interface is plotted against $\tau$, for variations in spike tip drag. The parameters are as described in the text with $\psi=1$ (solid), 2 (long-dashed), and 5 (short-dashed). The thin, black short-dashed line is the shock position.

(A color version of this figure is available in the online journal.)

One can then solve Equation (10) numerically, using the same treatment of the expansion that was used above for the linear theory. Figure 8 shows the results for $\psi=1.5$ using the parameters given in the caption and initiating the buoyancy-drag calculation at $\tau=1.3$ when the spike amplitude has reached $7.1 \mu \mathrm{m}$, which is $10 \%$ of the initial modulation wavelength. One can see that the effect of the expansion is to nearly double the basic growth due to the instability. This is of the same order as previous estimates (Miles et al. 2004; Kuranz et al. 2009b, 2009a). One can also see that for this value of $\psi$ the spikes would extend about $2 / 3$ of the way from the mean interface to the shock at $\tau \sim 15$, which corresponds to an experimental time $t \sim 25 \mathrm{~ns}$. At this time, the spikes would trail the forward shock by about $100 \mu \mathrm{m}$.

\section{RESULTS AND DISCUSSION}

It is now finally possible to address the question that motivated this study, and ask how variations in spike tip drag affect spike penetration. This is done in Figure 9, for $\psi=1,2$, and 5. For $\psi=1$, corresponding to cylindrical spikes with no tip broadening, the computed spike position exceeds the shock position for the present reference model having $\alpha=0.8$. However, the spikes actually would not penetrate (much) beyond the shock. The present model does not evaluate the greatly increased drag that spikes extending through the shock would encounter. Such spikes would be expected to extend to the shock, and potentially to distort it. A very modest amount of increased drag makes a substantial difference in the computed spike penetration. When the effective radius of the tip is only twice that of the stem $(\psi=2)$, the spike is found to extend less than half the distance from the mean interface to the shock. It is again substantially shorter for $\psi=5$.

The polytropic index $\gamma$ and the shock position exponent $\alpha$ act independently in this model. Increasing $\gamma$ increases the shockto-interface distance, which more than doubles as $\gamma$ increases from 1.2 to 1.6. This is no surprise, as the immediate postshock compression decreases by a comparable amount. For the specific experimental data discussed above, these limits (1.2 and 1.6) are beyond those reasonably allowed by the data. The spike penetration decreases as $\gamma$ increases, although the decrease is typically not large. The net effect is that the spikes reach the shock more readily for smaller values of $\gamma$, which produce narrower shocked layers. 

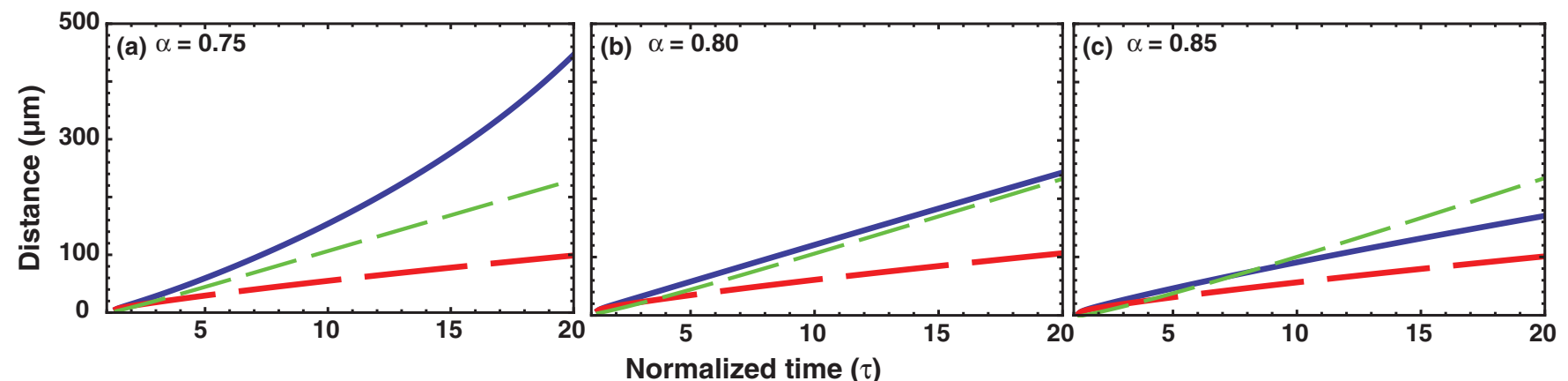

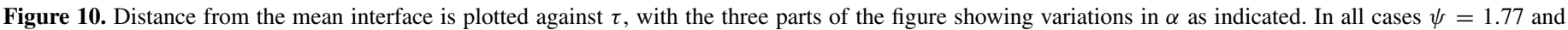

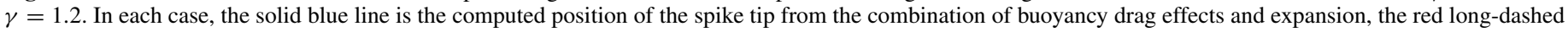
line is without expansion, and the green dashed line is the position of the shock.

(A color version of this figure is available in the online journal.)

Figure 10 shows the impact of changing $\alpha$ over the range of $0.75-0.85$. The three cases shown correspond to the three fits shown in Figure 1. Here again, the limits are somewhat beyond those consistent with all the evidence about the reference experiments. Note that both $t_{s}$ and $t_{1}$ are different in these three cases, so although the effect of the instability as such is the same in the normalized units of $\tau$, the physical time required to reach this degree of penetration differs. That said, one sees that increasing $\alpha$ corresponds to decreased total spike penetration, although in the specific case shown the spikes are near the shock for all values of $\alpha$. (Note again that when the spikes are computed by this model to be beyond the shock, realistically that means they are at the shock.) The same trend occurs when the spikes do not reach the shock, but the variation in spike penetration with $\alpha$ is modest.

Thus, we see that the spike penetration distance depends very strongly upon the shape (specifically the effective projected area) of the spike tips. Within the context of the parameters used here for our primary example, one would expect narrow spikes to always reach the forward shock in BWD instabilities. If this applied directly to the experiments that have seen narrow spikes (Kuranz et al. 2009a, 2010), the question would not be why sometimes the spikes reach the shock, but rather why they do not always do so. However, it would be overreaching to draw such a specific conclusion from the present approximate model of the material properties and behavior. Recalling that different simulations of the same problem find considerable variations in spike morphology, it would appear that the amount of spike penetration found in simulations is significantly uncertain.

As the calculation presented above is planar, some remarks are in order regarding the dynamics of spherical expansions, including core-collapse SNe and young SNRs. These systems produce far more expansion than planar systems do, so that the spikes present at late times have a length that was almost entirely produced by expansion. However, it takes some growth of structure to produce a spike that can be stretched by the expansion. An implication of Equation (10) is that early structure growth dominates the later spike length because it is this structure that is stretched. This in turn implies that the degree of RM vorticity deposition is central to the long-term behavior, since this drives the early structure growth. We note that core-collapse SN explosion modeling has yet to include any systematic evaluation of how modulations at composition interfaces may affect the subsequent structure, and that this is a large lever.
Also in the context of astrophysics, the preponderance of simulations using the piecewise parabolic method (PPM) should not blind one to the fact that this is only one possible method. On the one hand, in may be that PPM may be correct regarding the spike tip dynamics. On the other hand, it may be that no present method calculates the spike tip dynamics accurately. If, for example, it turned out that the PPM method produces unrealistically fat spike tips, then the challenge of successfully modeling SN 1987A (Hammer et al. 2010) would be greatly reduced.

\section{CONCLUSION}

In the present work I undertook to address a simple question in the context of BWD instabilities. The context was the penetration of spikes of dense material into a region of less dense material in consequence of the passage of a blast wave. The question was what range of effects spike tip broadening could have on the penetration of the spikes produced by such instabilities when buoyancy causes the (RT) amplification of structure initially produced by (RM) vorticity deposition when the shock front in the blast wave encounters an interface. The approach was to examine the evolution of a single 3D mode, which developed over time into independent spikes. This type of dynamics has been present in a number of laboratory experiments and could be present in astrophysical contexts where a surface is perturbed by a well-defined local modulation. The question of interest had to be addressed in several steps.

In the common geometry of experiments and core-collapse $\mathrm{SNe}$, where a shock front encounters an interface at which the density decreases, any modulations of the interface are inverted and stretched by the immediate rarefaction of the dense material while also being shortened by shock compression. Vorticity is also deposited across the interface. These elements were addressed using a combination of standard RM theory and basic fluid dynamics. This analysis revealed that the behavior of the perturbation during the shock passage through it has a significant impact on the post-shock state, creating an immediate reversal of the phase of the perturbation for typical conditions. Prior work on the RM process has often failed to pay attention to this issue. The interface decelerates after the shock front leaves it. It is useful to treat this deceleration as characterized by a power law in time, enabling one to find the corresponding, selfsimilar structure of the plasma properties between the shock wave and the interface. Such a power-law model also provided 
a reasonable, although far from unique, description of some experiments whose results were used as a reference case here.

During the interface deceleration, two things occur simultaneously-buoyancy causes RT growth while the structure present is stretched by the ongoing rarefaction of the post-shock material. Fortunately, these two effects act additively, so one can evaluate them independently in formulating a differential equation for the spike growth. Unfortunately, the stretching is not accurately represented by any simple model and depends on the specific history of spike length, so one is fairly much stuck with using numerical evaluations of the combined effects. I undertook this first for the linear phase of RT growth and then for the nonlinear phase in which the principal forces are those of buoyancy and drag acting on established spikes. The calculation ignored any potential intermediate phase in which the modulated interface may have developed a more complex modal structure before the spikes began to experience significant drag.

To address the question of interest, it was necessary to account for the variations in drag that presumably correspond to differing spike tip widths. I did this in the context of the buoyancy-drag dynamics. The drag was characterized by a parameter, $\psi$, that is approximately equal to the ratio of the spike tip diameter to the spike-stem diameter, although in full detail it will be modified by the impact of the shape of the spike tip on the drag and by the relative volumes of the spike stem and spike tip. For a given set of parameters, characterizing the initial post-shock modulations, the long-term shock deceleration, the polytropic index, and the relative size of the drag, it was then possible to evaluate the total spike penetration to compare this with the shock position.

The results showed that the amount of spike penetration depends very strongly upon the relative amount of drag. Narrow spikes, such as have been observed in some experiments, would often be expected to penetrate to the forward shock. Spikes having noticeable spike tip broadening would rarely if ever be expected to reach the forward shock, but the actual degree of penetration would be quite sensitive to the actual spike tip properties. Since the spreading of the spike tip is a very subsonic process, small effects either in reality-such as modest magnetic fields (Kuranz et al. 2010; Fryxell et al. 2010) - or in simulations_-such as vorticity generation or dissipation by the numerics - can readily change the result. It is significant but unfortunate that the calculation of subsonic flows affected substantially by vorticity, which produces the spike tip broadening, turns out to have such leverage on the large-scale dynamics. The implication is that one needs to find a way to validate these codes, against relevant and adequately diagnosed experiments, if one is to be able to believe that they correctly evaluate the degree of spike penetration and the consequent long-term implications for structure of astrophysical objects.

The author acknowledges useful discussions of the linear theory with Igor Sokolov, discussions of issues associated with spike penetration with Tomek Plewa, Bruce Fryxell, Aaron Miles, and Carolyn Kuranz, and good discussions and hospitality by Claire Michaut and Serge Bouquet. The author also thanks the referee whose thoughtful remarks stimulated improvements in the paper. Support for this research was provided by the Observatoire de Meudon, by the US DOE NNSA under the Predictive Sci. Academic Alliance Program by grant DE-FC52-08NA28616, the Stewardship Sci. Academic
Alliances program by grant DE-FG52-04NA00064, and the Nat. Laser User Facility by grant DE-FG0300SF22021.

\section{REFERENCES}

Arnett, D., Fryxell, B., \& Muller, E. 1989, ApJ, 341, L63

Betti, R., \& Sanz, J. 2006, Phys. Rev. Lett., 97, 205002

Cabot, W. 2006, Phys. Fluids, 18, 045101

Calder, A., Fryxell, B., Plewa, T., et al. 2002, ApJS, 143, 201

Chandrasekhar, S. 1961, Hydrodynamic and Hydromagnetic Stability (New York: Dover)

Chevalier, R. 1976, ApJ, 207, 872

Chevalier, R. 1982, ApJ, 258, 790

Chevalier, R., Blondin, J., \& Emmering, R. 1992, ApJ, 392, 118

Dimonte, G. 2000, Phys. Plasmas, 7, 2255

Dimonte, G., Frerking, C., Schneider, M., \& Remington, B. 1996, Phys. Plasmas, 3,614

Dimonte, G., \& Ramaprabhu, P. 2010, Phys. Fluids, 22, 014104

Dimonte, G., \& Remington, B. 1993, Phys. Rev. Lett., 70, 1806

Dimonte, G., \& Schneider, M. 1996, Phys. Rev. E, 54, 3740

Dimonte, G., Youngs, D. L., Dimits, A., et al. 2004, Phys. Fluids, 16, 1668

Doss, F. 2011, PhD thesis, Univ. Michigan

Drake, R. 2005, Plasma Phys. Control. Fusion, 417, B419

Drake, R. 2006, High Energy Density Physics: Fundamentals, Inertial Fusion and Experimental Astrophysics (Berlin: Springer)

Drake, R., Leibrandt, D., Harding, E., et al. 2004, Phys. Plasmas, 11, 2829

Fryxell, B., Kuranz, C. C., Drake, R. P., et al. 2010, High Energy Density Physics, 6, 162

Fryxell, B., Muller, E., \& Arnett, D. 1991, ApJ, 367, 619

Glendinning, S. G., Bolstad, J., Braun, D. G., et al. 2003, Phys. Plasmas, 10, 1931

Glimm, J., Li, X. L., \& Lin, A.-D. 2002, Acta Math. Applacatae Sin., 18, 1

Hammer, N. J., Janka, H. T., \& Muller, E. 2010, ApJ, 714, 1371

Hester, J. J., Scowen, P. A., Sankrit, R., et al. 1996, ApJ, 456, 225

Hughes, J., Rakowski, C., \& Decourchelle, A. 2000, ApJ, 543, L61

Jacobs, J. W., \& Catton, I. 1988, J. Fluid Mech., 187, 353

Jacobs, J. W., \& Sheeley, J. M. 1996, Phys. Fluids, 8, 405

Kifonidis, K., Plewa, T., Janka, H.-T., \& Muller, E. 2000, ApJ, 531, L123

Kifonidis, K., Plewa, T., Janka, H.-T., \& Muller, E. 2003, A\&A, 408, 621

Kifonidis, K., Plewa, T., Sheck, L., Janka, H.-T., \& Muller, E. 2006, A\&A, 457, 963

Kuranz, C., Drake, R., Grosskopf, M., et al. 2009a, Phys. Plasmas, 16, 156310

Kuranz, C., Drake, R., Grosskopf, M., et al. 2010, Phys. Plasmas, 17, 052709

Kuranz, C., Drake, R., Harding, E., et al. 2009b, ApJ, 696, 749

Landau, L., \& Lifshitz, E. 1987, Course in Theoretical Physics, Vol. 6, Fluid Mechanics (2nd ed; Oxford: Pergamon)

Liang, E., \& Keilty, K. 2000, ApJ, 533, 890

Meshkov, E. 1969, Sov. Fluid Dyn., 4, 101

Meyer, K., \& Blewett, P. 1972, Phys. Fluids, 15, 753

Miles, A. 2009, ApJ, 696, 498

Miles, A. R., Braun, D. G., Edwards, M. J., et al. 2004, Phys. Plasmas, 11, 3631

Mitchner, M., \& Landshoff, R. K. M. 1964, Phys. Fluids, 7, 862

Muller, E., Fryxell, B., \& Arnett, D. 1991, A\&A, 251, 505

Parker, E. 1963, Interplanetary Dynamical Processes (New York: Interscience)

Plesset, M. S., \& Hsieh, D. Y. 1964, Phys. Fluids, 7, 1099

Ramaprabhu, P., \& Dimonte, G. 2005, Phys. Rev. E, 71, 036314

Ramaprabhu, P., Dimonte, G., Young, Y. N., Calder, A. C., \& Fryxell, B. 2006, Phys. Rev. E, 74, 066308

Rayleigh, L. 1900, Scientific Papers II (Cambridge: Cambridge Univ. Press), 200

Remington, B. A., Weber, S. V., Marinak, M. M., et al. 1995, Phys. Plasmas, 2, 241

Richtmyer, R. 1960, Commun. Pure Appl. Math, 13, 297

Ryutov, D., Drake, R., Kane, J., et al. 1999, ApJ, 518, 821

Taylor, S. G. 1950, Proc. R. Soc. A, 201, 192

Vishniac, E. 1983, ApJ, 274, 152

Vishniac, E., \& Ryu, D. 1989, ApJ, 337, 917

Warren, J. S., Hughes, J. P., Badenes, C., et al. 2005, ApJ, 634, 376

Wei, T., \& Livescu, D. 2011, Phys. Fluids, submitted

Wilkinson, J. P., \& Jacobs, J. W. 2007, Phys. Fluids, 19, 124102

Wouchuk, J. G. 2001, Phys. Plasmas, 8, 2890 